\title{
Brillouin optical time-domain analysis sensor with amplification of pump pulses and tolerant to non-local effects
}

\author{
Juan José Mompó, Javier Urricelqui and Alayn Loayssa* \\ Departamento de Ingeniería Eléctrica y Electrónica, Universidad Pública de Navarra \\ Campus de Arrosadía s/n, 31006, Pamplona, Spain
}

\begin{abstract}
We demonstrate a simple technique to provide conventional Brillouin optical time-domain analysis sensor with compensation for pump pulse attenuation and tolerance to non-local effects. The technique is based on operating the sensor in loss configuration so that energy is transferred from the probe wave to the pump pulse that becomes amplified as it counter-propagates with the probe wave. Furthermore, the optical frequency of the probe wave is modulated along the fiber so that the pump pulse experiences a flat gain spectrum that equally amplifies all the spectral components of the pulse, hence, preventing distortion. The method is experimentally demonstrated in a 100-km fiber link, obtaining a measurement uncertainty of $1 \mathrm{MHz}$ at the worst-contrast position.
\end{abstract}

Keywords: Stimulated Brillouin scattering, Brillouin optical time-domain analysis, strain and temperature measurements

\section{INTRODUCTION}

In the last years, Brillouin optical time-domain analysis (BOTDA) sensors have been used to monitor structures such as oil and gas pipelines or high voltage transmission lines. All of these applications have a common need for large monitoring distances. However, the sensing range of BOTDA sensors is essentially limited by pump attenuation in the optical fiber. Moreover, this limitation can not be compensated by indefinitely increasing the pulse power at the input of the fiber because of the onset of modulation instability (MI) and other nonlinear effects that degrades the measurements. A possible solution is to amplify the pump pulses by using distributed Raman amplification, but this requires high pump power in the fiber (of the order of watts) and increase the detected intensity noise degrading the signal-to-noise ratio (SNR). ${ }^{1}$

We have recently presented an alternative solution based on amplifying the pump pulses using a distributed Brillouin amplifier (DBA). ${ }^{2}$ This technique is based on injecting in the fiber another laser source acting as DBA pump that amplifies the pulsed signal and does not increase the noise on the detected probe wave. In addition, we have been working lately on the compensation of the other two factors that limit the probe wave power and hence, the sensing range: non-local effects and Brillouin threshold of the fiber. In order to simultaneously compensate both factors, we have introduced a dual-probe-sideband BOTDA setup in which the optical frequency of the probe waves is modulated along the fiber. ${ }^{3}$

In this work, we have built upon our previous contributions by introducing a simple modification to conventional BOTDA sensors that simultaneously provides compensation of pulse attenuation along the fiber and allows to increase the power of probe wave by compensating non-local effects and the intensity noise induced when injecting a probe power larger than the Brillouin threshold of the fiber. The technique is based on operating the sensor in loss configuration, so that energy is transferred from the probe wave to the pump pulse, and on modulating the optical frequency of the probe wave along the fiber.

E-mail: alayn.loayssa@unavarra.es 


\section{DESCRIPTION OF THE TECHNIQUE}

Fig. 1(a) highlights the fundamentals of the technique in which two optical waves are used as in any conventional BOTDA sensor: the probe wave and the pulsed signal. As it is shown, the BOTDA sensor is set in a loss-based configuration so that the pump pulses generate a loss spectrum for the counter-propagating probe wave at every position along the fiber. Simultaneously, the probe wave also transfers energy to the pump as they interact along the fiber. The only modification is that the probe wave optical frequency is modulated with a saw-tooth shape so that at every location of the fiber, as it is depicted in Fig. 1(a), the Lorentzian gain spectrum experienced by the pulses is slightly offset in frequency. This makes the center optical frequency of the Brillouin gain spectra experienced by the pump pulses to vary along the fiber as it meets the optical-frequency-modulated probe wave front, so that the pulse experiences a broad total integrated gain spectrum. This net gain spectrum can be made to have a flat frequency response with a judicious choice of modulation shape, frequency and peak deviation. ${ }^{4}$ Furthermore, the frequency modulation is synchronized to the pulsed signal so that a sequence of pulses experience the same optical frequency of the probe wave at any given location.

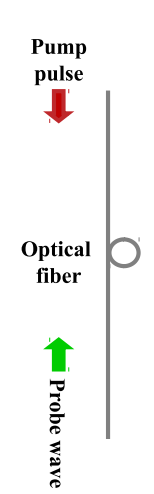

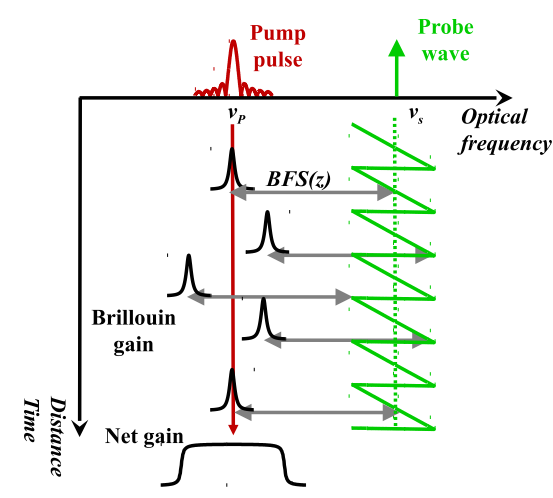

(a)

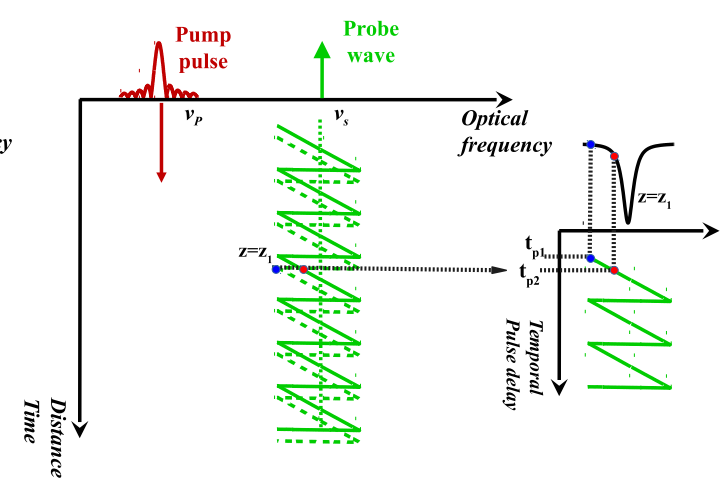

(b)

Figure 1. (a) Spectra of the optical waves present in the optical fiber and (b) frequency scanning method based on the temporal delay between probe and pump waves.

In addition, the frequency modulation of the probe wave is also employed to perform the frequency scan of the Brillouin spectra distribution instead of using the conventional frequency swept. Fig. 1(b) schematically depicts the method to scan the loss spectra at every location of the fiber simultaneously, which simply requires to change the temporal delay between the probe wave modulation and the pump pulse. ${ }^{3}$ As shown in the figure, the modification of the delay in steps displaces the z-location of the saw-tooth so that, after a total delay equivalent to the period of the saw-tooth all frequencies within the peak-to-peak deviation of the frequency modulation have been scanned at all locations in the fiber. Notice that there is no measurement time penalty in this setup compared to a conventional frequency-swept BOTDA and hence, both systems require an identical number of measurements to characterize the full Brillouin spectrum for every position of the fiber.

\section{EXPERIMENTAL RESULTS}

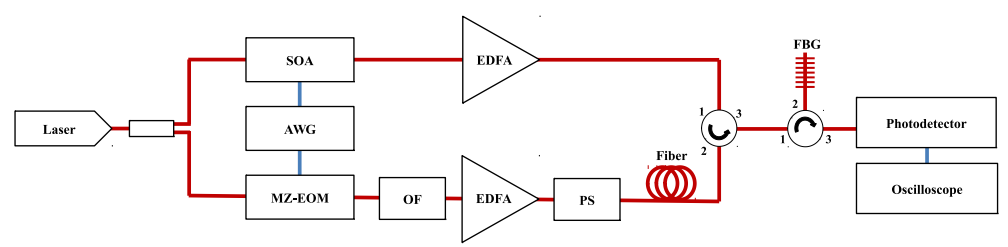

Figure 2. Experimental setup for the BOTDA sensor based on frequency modulation of the probe wave.

Fig. 2 schematically depicts the setup used to demonstrate our technique. We modify the conventional BOTDA by adding an arbitrary wave generator (AWG) to frequency modulate the microwave signal applied to the Mach-Zehnder electro-optic modulator (MZ-EOM), biased at minimum transmission used to generate the 
probe wave. The AWG provides a FM microwave signal whose instantaneous frequency varies around the average Brillouin frequency shift (BFS) of the fiber following a saw-tooth shape with a period of 12.5 microseconds and peak-to-peak frequency deviation of $200 \mathrm{MHz}$. This frequency modulation signal is synchronized to the electrical pulse signal applied to the semiconductor optical amplifier (SOA) to pulse the signal in the upper branch, which is generated on the same AWG. After the MZ-EOM, the lower-frequency sideband is filtered out by an optical filter (OF). Finally, the probe wave is boosted to $9 \mathrm{dBm}$ using an erbium-doped fiber amplifier (EDFA) and its polarization state is randomized using a polarization scrambler (PS) before been injected into 100-km of G.652 sensing fiber. In the upper branch, the pulse is amplified in an EDFA to a peak power of $70 \mathrm{~mW}$ and launched into the fiber.

Fig. 3 compares the distribution of the Brillouin spectra measured along the fiber with a dual-probe-sideband BOTDA $^{3}$ and for the new BOTDA with amplification of the pulse. For the dual-probe BOTDA, the amplitude of the Brillouin spectra decays exponentially due to the attenuation of the pump pulse as it propagates through the fiber. In contrast, in our system, the amplitude initially decays but starts to recover at a distance of around $30 \mathrm{~km}$ from the pump input, as it can be seen more clearly in Fig. 4(a).

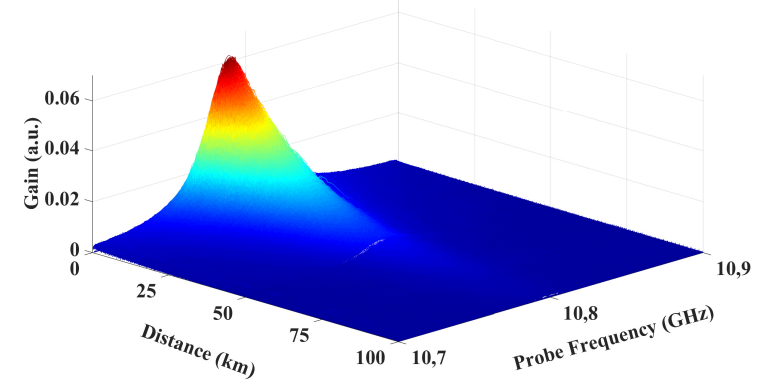

(a)

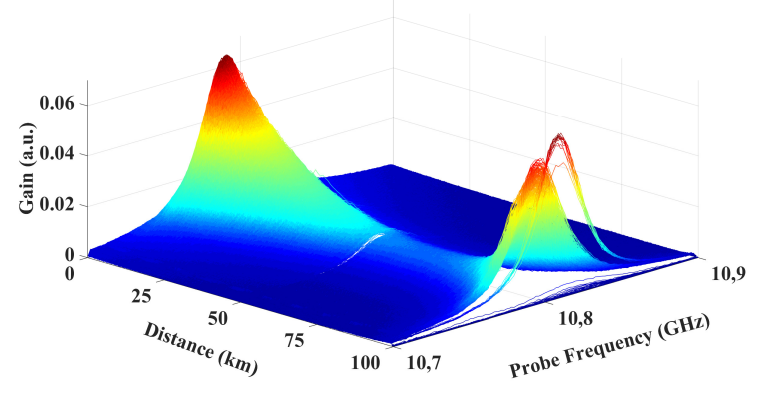

(b)

Figure 3. Brillouin gain distribution measured with (a) dual-probe-sideband BOTDA sensor using frequency modulation of the probe wave and (b) novel BOTDA with pulse amplification. A pulse of $45 \mathrm{~ns}$ was deployed in both measurements.

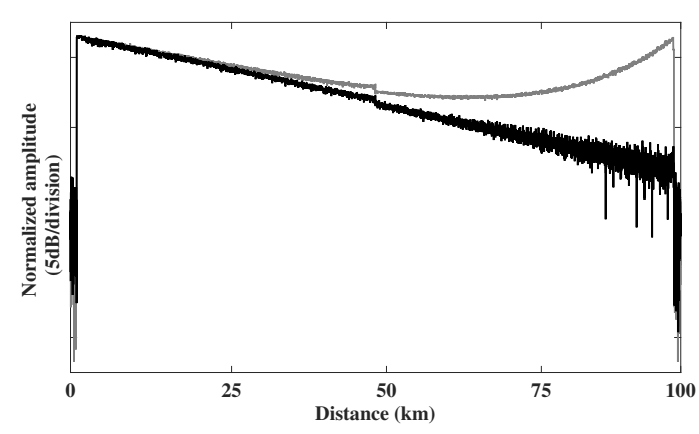

(a)

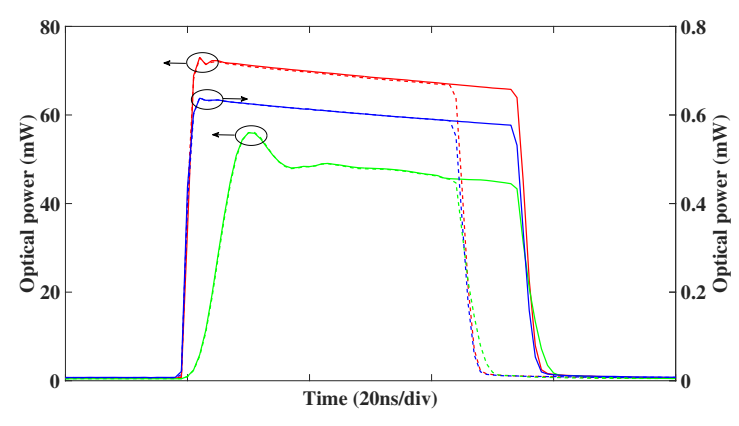

(b)

Figure 4. (a) Mesured traces of dual-probe-sideband BOTDA (black line) and BOTDA with pulse gain (grey line) using 45-ns pulse duration. (b) Amplification of pump pulses with 45-ns (dashed line) and 55-ns (solid line) duration. Pulses at the input of the fiber (red line), output of the fiber without gain (blue line) and amplified pulses (green line).

Fig. 4(b) shows the amplification of pulses in the fiber. The pulses at the input of the fiber and at the output with and without gain are compared for pulses with 45-ns and 55-ns durations. The pulses are amplified by $19.62 \mathrm{~dB}$, compensating the fiber link attenuation. Notice that the amplified pulse has its rise-time smoothed by the exponential growth of the acoustic waves as it meets the probe wave. This is an effect that we have previously observed and that makes the pulse asymmetrical in the time-domain, which can lead to a BFS error when self-phase modulation (SPM) comes into play. ${ }^{2}$ However, this potential error can be suppressed by 
deploying differential pulse-width pair (DPP) measurements. ${ }^{5}$

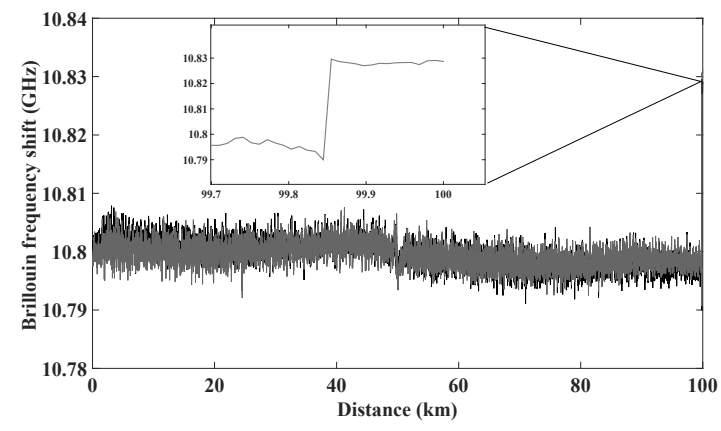

(a)

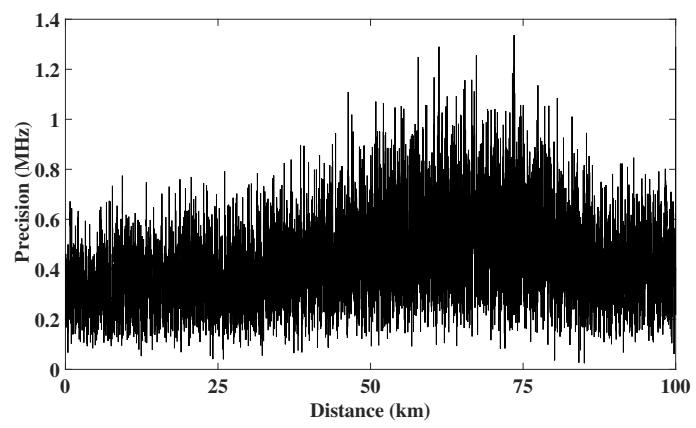

(b)

Figure 5. (a) Measured BFS distribution along the fiber and (inset) in the hotspot. (b) Precision of the measurement.

Fig. 5(a) displays the measured BFS distribution in the fiber using DPP technique with two pulses of 45 and $55 \mathrm{~ns}$. Two measurements were sequentially performed with the fiber inputs swapped so as to make sure that the BFS distribution is identical independently of which end is used to inject the probe and pump waves and hence that SPM or non-local effects are not introducing any significant errors. In addition, the inset of the figure depicts the BFS in the last $140 \mathrm{~m}$ of the fiber which were kept at $55^{\circ} \mathrm{C}$ using a climatic chamber. The observed BFS change in this area is consistent with the temperature change with respect to the rest of the fiber. Finally, Fig. 5(b) shows the measurement uncertainty along the fiber calculated as the standard deviation of 5 consecutive measurements. As it was expected the measurement precision and the spectra amplitude in Fig. 3(b) are directly related. The areas with larger uncertainty coincide with lower spectra amplitude. All these measurements have been performed with 1500 averages of the traces.

\section{CONCLUSIONS}

In summary, we have demonstrated a BOTDA sensor that with minor alterations to the conventional setup is able to provide gain to the pump pulses and extend the measurement range up to $100 \mathrm{~km}$ with 1-m resolution. This is done without the need to add any amplification scheme, such as Raman amplification, that is not intrinsically present in the typical interaction of a BOTDA sensor.

\section{ACKNOWLEDGMENTS}

The author's wish to acknowledge the financial support from the Spanish Ministerio de Economía y Competitividad through project TEC2013-47264-C2-2-R, FEDER funds and the Universidad Pública de Navarra.

\section{REFERENCES}

1. X. Angulo-Vinuesa, S. Martín-López, J. Nuño, P. Corredera, J. Ania-Castañon, L. Thévenaz, and M. Gonzalez-Herraez, "Raman-assisted Brillouin distributed temperature sensor over $100 \mathrm{~km}$ featuring $2 \mathrm{~m}$ resolution and $1.2^{\circ} \mathrm{C}$ uncertainty," L. Lightw. Technol. 30, pp. 1060-1065, April 2012.

2. J. Urricelqui, M. Sagues, and A. Loayssa, "Brillouin optical time-domain analysis sensor assisted by Brillouin distributed amplification of pump pulses," Opt. Express 23, pp. 30448-30458, Nov 2015.

3. R. Ruiz-Lombera, J. Urricelqui, M. Sagues, J. Mirapeix, J. López-Higuera, and A. Loayssa, "Overcoming nonlocal effects and Brillouin threshold limitations in Brillouin optical time-domain sensors," Photonics Journal, IEEE 7, pp. 1-9, Dec 2015.

4. A. Zadok, A. Eyal, and M. Tur, "Gigahertz-wide optically reconfigurable filters using stimulated Brillouin scattering," L. Lightw. Technol. 25, pp. 2168-2174, Aug 2007.

5. W. Li, X. Bao, Y. Li, and L. Chen, "Differential pulse-width pair BOTDA for high spatial resolution sensing," Opt. Express 16, pp. 21616-21625, Dec 2008. 
Copyright 2015 Society of Photo Optical Instrumentation Engineers. One print or electronic copy may be made for personal use only. Systematic reproduction and distribution, duplication of any material in this paper for a fee or for commercial purposes, or modification of the content of the paper are prohibited. 